\title{
A Spontaneous Spinal Epidural Hematoma in a Hemodialysis Patient: A Rare Entity
}

\author{
Serpil Muge Deger ${ }^{1}$, Hakan Emmez ${ }^{2}$, Kenan Bahadirli ${ }^{3}$, Aydemir Kale ${ }^{3}$, \\ Fatma Ayerden Ebinc ${ }^{3}$, Melda Turkoglu ${ }^{4}$, Turgay Arinsoy ${ }^{4}$ and Sukru Sindel ${ }^{4}$
}

\begin{abstract}
Hemodialysis patients are at an increased risk of bleeding due to the platelet dysfunction caused by uremia and the use of anticoagulants during dialysis. Spontaneous spinal hematoma is a rare disorder as a complication in hemodialysis patients. Also it includes the hematoma secondary to coagulopathy, vascular malformation and hemorrhagic tumors. Here, we report the case of 77-year-old woman who presented with spinal cord compression due to spontaneous spinal epidural hematoma associated with hemodialysis. When an end-stage renal disease patient suffers from back pain and neurological deficits, the clinician should be alerted for the spontaneous spinal epidural hematoma as well as cerebrovascular events.
\end{abstract}

Key words: epidural hematoma, hemodialysis, neurosurgery

(Inter Med 48: 2115-2118, 2009)

(DOI: 10.2169/internalmedicine.48.2335)

\section{Introduction}

Cerebrovascular events, as well as cardiovascular diseases are important causes of morbidity and mortality in dialysis patients. Hemodialysis patients are at an increased risk of bleeding due to the platelet dysfunction caused by uremia and the use of anticoagulants during dialysis. Epidural hematoma is a neurosurgical emergency $(1,2)$ and it includes idiopathic hematomas or hematomas secondary to coagulopathy, tumor and vascular malformation (3). Although epidural hematomas seen in this group of patients are generally associated with head trauma (1), spontaneous spinal epidual hematoma (SSEH) can be seen as an uncommon event. To our knowledge there are few reports in the literature about spontaneous spinal epidural hematomas (SSEH) in dialysis patients. Here, we describe SSEH between C2 and $\mathrm{T} 9$ in a dialysis patient, presenting with neck and back pain and acute motor weakness in lower limbs and right upper limb.

\section{Case Report}

A 77-year-old woman with a diagnosis of end-stage renal disease (ESRD) as a consequence of hypertension was admitted to our emergency department with the sudden onset of cervical and back pain. She had been on hemodialysis routinely three times a week with standard bicarbonate for three years, her kt/v was 1.32 and during the hemodialysis $5,000 \mathrm{IU}$ heparin was used. In her dialysis her ultrafiltration volume was 2,400-2,500 $\mathrm{cc}$ and 2,500 $\mathrm{cc}$ in her last dialysis. A few hours after her admission parasthesia, weakness of the bilateral lower limbs and right upper limb developed. Clinical examination revealed a bilateral lower limb weakness (scored 2/5), right upper limb weakness (scored 0/5) without any sphincter disorder or any disorder of consciousness. There were no external signs of the head or spinal trauma. Her tension arterial was 130/80 and in her follow-up tension arterial measurements were changed from 100/60 to 130/80. While her cranial computed tomographic scan was normal, cervical and thoracal spinal MRI showed a dorsally located epidural lesion which was iso-hyperintense on T1-

\footnotetext{
${ }^{1}$ Department of Nephrology, Gazi University Faculty of Medicine, Ankara, Turkey, ${ }^{2}$ Department of Neurosurgey, Gazi University Fcaulty of Medicine, Ankara, Turkey, ${ }^{3}$ FMC Dialysis Center, Ankara, Turkey and ${ }^{4}$ Department of Intensive Care Unit, Gazi University Faculty of Medicine, Ankara, Turkey

Received for publication April 4, 2009; Accepted for publication July 21, 2009

Correspondence to Dr. Serpil Muge Deger, serpilmugedeger@yahoo.com
} 


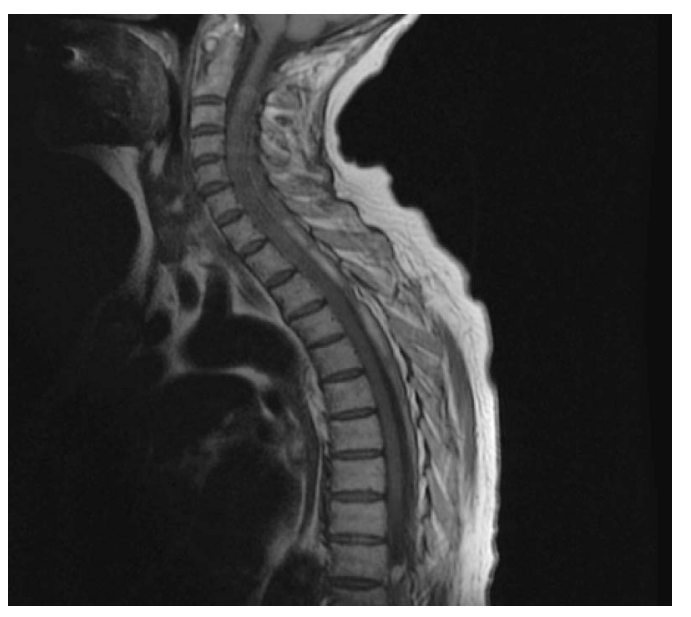

Figure 1a. Sagittal T1-weighted images showing iso-hyperintense posterior epidural lesion (hematoma) compressing the spinal cord between $\mathrm{C} 2$ and $\mathrm{T} 9$.

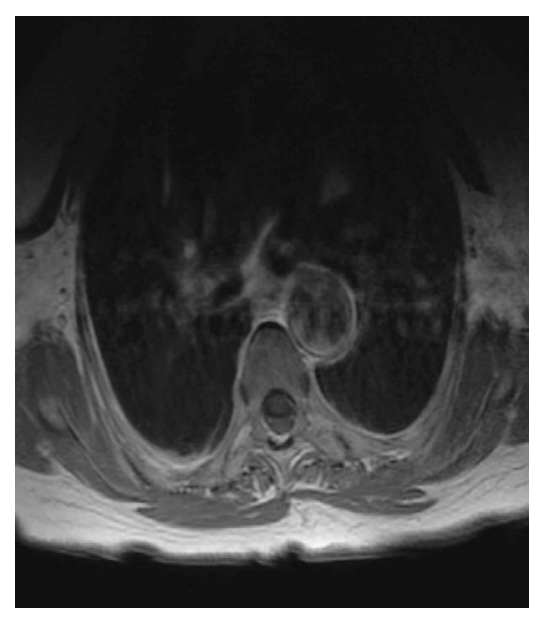

Figure 1b. Axial T1-weighted images showing spinal cord compression.

weighted images (Figs. 1a, 1b) and iso-hypointense on T2weighted images between $\mathrm{C} 2$ and T9 level (Figs. 2a, 2b) and also a marked compression of the spinal cord. Various laboratory tests were performed. Her blood urea nitrogen (BUN) level was $48 \mathrm{mg} / \mathrm{dl}$, serum creatinin concentration was $7.5 \mathrm{mg} / \mathrm{dl}$, partial thromboplastin time was $22.1 / \mathrm{sec}$, prothrombin time was $11 / \mathrm{sec}$, platelet count was 145,000 $\mathrm{mm}^{3}$ and her bleeding time was 4 minutes. Her last dialysis had been performed two dayspreviously. She had been receiving medications including antihypertensive agents (atenolol, isosorbid-5-mononitrate) and atorvastatin. Immediately, the patient underwent $\mathrm{C} 4, \mathrm{C} 5$ total laminectomy, C7T3-T5 left hemilaminectomy and evacuation of the epidural hematoma. There was no evidence of vascular anomaly or neoplasia. Postoperatively, the patient had a good recovery in lower limbs (4/5 paraparesis) and poor recovery in the right upper limb. She was discharged in stable condition.

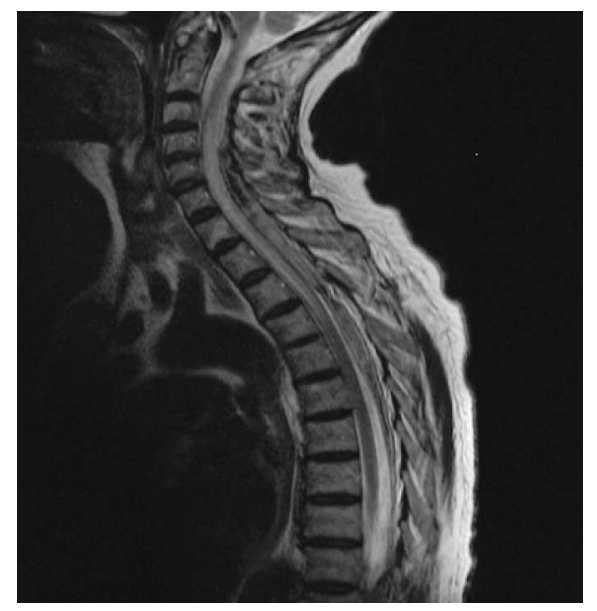

Figure 2a. Sagittal T2-weighted images showing an iso-hypointense posterior epidural lesion (hematoma) compressing the spinal cord between $\mathrm{C} 2$ and $\mathrm{T} 9$.

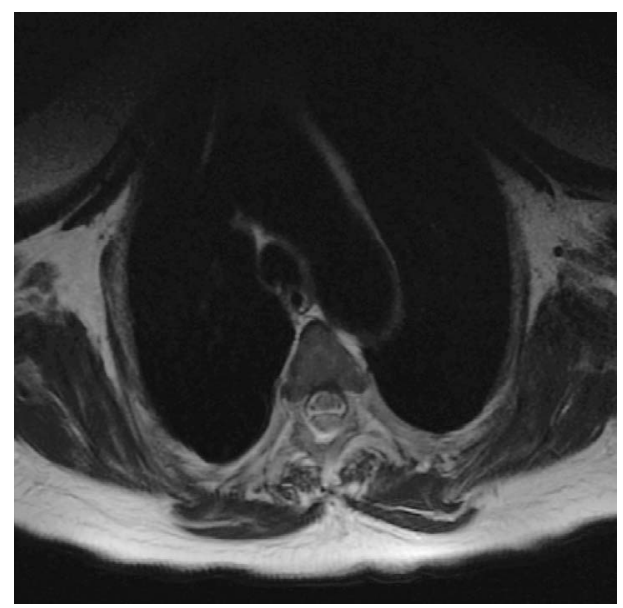

Figure 2b. Axial T2-weighted images showing the spinal cord and tecal sac compression.

\section{Discussion}

Although epidural hematomas usually have traumatic origin (1), spontaneous spinal epidural hematoma, which is a rare condition, develops spontaneously in the absence of the any iatrogenic procedure like lumbar puncture and trauma. Also spontanous spinal epidural hematoma includes hematoma secondary to coagulopathy, vascular malformation and hemorrhagic tumors (3). The most affected regions in SSEH are the cervicothoracic and thoracolumbar parts of the spine (4).

The most important pathophysiological finding in this condition is venous bleeding. The increase in intraabdominal or intrathoracic pressure to any reason such as pregnancy causes rupture in epidural veins with or without valves resulting in SSEH. Also abnormal coagulation may trigger venous hemorrhage $(3,5)$. Although most of the researchers have accepted this hypothesis, some authors have attributed 
Table 1. The Case Reports of the Spontaneous Spinal Cranial Epidural Hematoma

\begin{tabular}{l|lll}
\hline Author & Patient & Location & Hemodialysis \\
\hline Ziyal IM et al (6) & 47 year old female & Spinal & Yes \\
Sulowicz W et al (9) & 20 year old female & Cranial & Yes \\
Shimokawa S et al (10) & 54 year old male & Cranial & Yes \\
Takahashi K et al (5) & 67 year old female & Spinal & Yes \\
Sung J et al & 61 year old male & Spinal & Yes \\
\hline Shahlaie K et al (8) & 16 year old male & Cranial & No \\
\hline
\end{tabular}

SSEH to arterial bleeding (3). Further studies are needed to delineate the precise pathogenesis of this condition.

There are several reports in the literature about SSEH and spontaneous cranial epidural hematomas in patients with end-stage renal disease (ESRD) receiving hemodialysis (5-11) (Table 1). Uremic bleeding caused by platelet dysfunction is a major cause of the morbidity and the mortality in ESRD patients. The pathogenesis of the platelet dysfunction in uremia includes reduction in intracellular adenosine diphosphate and serotonin, increased nitric oxide production, reduced total Gplb content, reduced GpIIb/IIIa levels and abnormal platelet adherence because of the uremic toxins (12). Also in hemodialysis patients, intravenous heparin administration during dialysis transiently causes the abnormalities in hemostasis (12). Kurokawa et al described an other mechanism of SSEH, in that the changes in the intracranial pressure during hemodilaysis contribute to the vascular damage and hemorrhage in dialysis patients $(10,13)$.

Although some investigators found no statistically significant relation, hypertension can be responsible for the development of the $\operatorname{SSEH}(7,8)$. To date several mechanisms have been hypothized for the development of SSEH but still its etiology remains controversial. In the present patient, there was no trauma or iatrogenic procedure history and in her physical examination no trauma signs were seen. Her arterial tension was normal. No bleeding disorder was found in laboratory assesment and in surgery no abnormal vessels were noted. In the light of the all findings, the heparin administration on hemodialysis and uremic bleeding abnormalities seemed to be responsible factors in the development of SSEH.

The most common clinical symptoms of this condition include the sudden onset of the back pain followed by neurological deficits, depending on the levels of the lesion. In some cases sphincter disorders have developed (2). In this case, she had the sudden onset of the cervical and low back pain and a few hours later parasthesia with weakness of the limbs developed similar to previously reported cases.

For the diagnosis, to determine the localisation of bleeding and to date the hematoma, MRI is the most beneficial imaging examination (3). In the present case, the hematoma was diagnosed by the cervical and thoracic MRI and the C2-T9 epidural lesion was demonstrated as hyperintense on T1 and hypointense on T2-weighted images. To our knowledge there is one report in the literature with an affected 15 segment of the spine (6) and the present case is the second case of multilevel lesions.

One of the treatment options for SSEH is surgery. After correction of the clotting disorders, decompressive laminectomy and evacuation of the hematoma and the exploration of the vessel anomaly should be performed. Cervical spinal epidural hematomas in particular are life threatening and often fatal without surgery $(2,3,5,8)$. But there are several reports in the literature that include conservative treatment $(2,3,14)$. Although these researchers reported that the patients with moderate symptoms, spontaneous improvement and minor compression on the MRI can be followed by conservative treatment, currently, early surgical intervention is mandatory in most of the cases, as in the present case $(2,3,14)$. Further, the results of the conservative management were poor, especially in cervical lesions. (15).

In conclusion, SSEH is a rare disorder and early surgery is the best treatment. For the definitive diagnosis MRI should be done. ESRD patients have a high risk for the development of hemorrhage because of heparin administration during dialysis, hypertension and uremic bleeding disorder. Therefore, when an ESRD patient suffers from back pain, neurological deficits, the clinician should be alerted for SSEH as well as cerebrovascular events.

\section{References}

1. Ng WH, Yeo TT, Seow WT. Nontraumatic spontaneous acute epidural haematoma-report of two cases and review of the literature. J Clin Neurosci 11: 791-793, 2004.

2. Ananthababu PS, Anbuselvam M, Radhakrishnan MK. Spontaneous spinal epidural haematoma: report of two cases and review of the literature. J Clin Neurosci 12: 90-92, 2005.

3. Lonjon MM, Paquis P, Chanalet S, Grellier P. Non-traumatic spinal epidural hematoma: Report of four cases and review of the literature. Neurosurgery 41: 483-487, 1997.
4. Groen RJM, Ponssen H. The spontaneous spinal epidural hematoma: A study of the etiology. J Neurol Sci 98: 121-138, 1990.

5. Takahashi K, Fumihiko K, Tayama H, Satomi A, Akizawa T, Ideura T. A case of acute spontaneous epidural haematoma in a chronic renal failure patient undergoing haemodialysis: successful outcome with surgical management. Nephrol Dial Transplant 14: 2499-2501, 1999.

6. Ziyal I, Aydin S, Inci S, Sahin A, Ozgen T. Multilevel acute spinal epidural hematoma in a patient with chronic renal failure. Neurol 
Med Chir (Tokyo) 43: 409-412, 2003.

7. Sung J, Hsieh CC, Yu CY, Huang JJ. Acute spontaneous spinal epidural hematoma in a hamodilaysis patient with a bleeding tendency. Nephron 91: 358-360, 2002.

8. Shahlaie K, Fox A, Butani L, Boggan JE. Spontaneous epidural hemorrhage in chronic renal failure. Pediatr Nephrol 19: 1168$1172,2004$.

9. Sulowicz W, Kraśniak A, Gościński I, et al. Long-term good results of surgical treatment for spontaneous epi-and subdural hematoma in a female patient on maintenance hemodialysis. Przegl Lek 57: 764-765, 2000 (in Polish).

10. Shimokawa $S$, Hayashi $T$, Anegawa $S$, et al. Spontaneous epidural hematoma in a patient undergoing hemodialysis: a case report. No To Shinkei 55: 163-166, 2003 (in Japanese).

11. Sung J, Hsieh CC, Yu CY, et al. Acute spontaneous spinal epidural hematoma in a hemodialysis patient with a bleeding ten- dency. Nephron 91: 358-360, 2002.

12. Hörl W. Other blood and immune disorders in chronic kidney disease. In: Comprehensive Clinical Nephrology. 3rd ed. Feehaly J, Floege J, Johnson JR, et al, Eds. Mosby, Philadelphia, 2007: 861867.

13. Kurokawa Y, Suzuki S, Hashi K, et al. Elevation of intracranial pressure during hemodialysis- continuous measurement of cerebrospinal fluid presure in a patient with acoustic neurinoma. No To Shinkei 42: 569-573, 1990 (in Japanese).

14. Clarke DB, Betrand G, Tampieri D. Spontaneous spinal epidural hematoma causing paraplegia: resolution and recovery without surgical decompression. Neurosurgery 30: 108-111, 1992.

15. Duffill J, Sparrow OC, Millar J, Barker CS. Can spontaneous spinal epidural haematoma be managed safely without operation? A report of four cases. J Neurol Neurosurg Psychiatry 69: 816-819, 2000 .

(C) 2009 The Japanese Society of Internal Medicine http://www.naika.or.jp/imindex.html 